Systematic Review

\title{
The Effectiveness of Interventions to Reduce the Nurses' Distractions during Medication Administration: A Systematic Review
}

\author{
Chandra Apriadi Panduwal and E. C. Bilaut
}

Regional General Hospital (RSUD) Prof. Dr. W. Z. Johannes Kupang, Nusa Tenggara Timur, Indonesia

\begin{abstract}
Introduction: Nurses constitute the largest group of health professionals who work in the hospital setting and most of the medications in the setting are administered by nurses. Errors related to medication conducted by a nurse frequently occur during medication administration. Interruptions or distractions during medication administration have been identified as significant contributory factors to medication administration errors (MAEs).

Methods: This systematic review critically reviewed the evidence of the effectiveness of the interventions that aim to reduce nurse interruptions or distractions during medication administration. The search for the relevant literature was conducted in August 2018 using three databases; Medline, Cinahl and Embase.

Results: Nineteen full text articles were retrieved and reviewed, and 7 articles were included in this review. Five of these studies showed evidence of a reduction in the interruption or distraction rates in post-intervention measurements, while 4 studies reported a statistically significant reduction in the interruption or distraction rates, with $\mathrm{p}$ values between 0.0005 and 0.002 .

Conclusion: There was limited evidence available to support the effectiveness of the interventions in terms of either reducing the interruptions or distractions of the nurses during the medication administration or in terms of reducing the medication administration error rates.
\end{abstract}

\section{ARTICLE HISTORY}

Received: Dec 26, 2019

Accepted: Dec 31, 2019

\section{KEYWORDS}

nurse's distraction; medication; administration

\section{CONTACT}

Chandra Apriadi Panduwal $\Delta$

ammachandranusantara@gmail.com $\doteq$ Regional General Hospital (RSUD) Prof. Dr. W. Z. Johannes Kupang, Nusa Tenggara Timur, Indonesia

Cite this as: Panduwal, C. A., \& Bilaut, E. C. (2019). The Effectiveness of Interventions to Reduce the Nurses' Distractions during Medication Administration: A Systematic Review.Jurnal Ners, 14(3si), 132-140. doi:http://dx.doi.org/10.20473/in.v14i3(si).17048

\section{INTRODUCTION}

Medication administration is the most common intervention for hospitalized patients to improve health conditions and outcomes. However, medication administration within hospitals has become a prominent source of medication error and adverse events in a hospital and these interventions have caused serious harm compared to other medication errors. (Australian Commission on Safety and Quality in Health Care, 2012; Picone et al., 2008; Raban \& Westbrook, 2014; "Strategies to reduce medication errors with reference to older adults.," 2006)In fact, Medication Administration Errors (MAEs) are preventable events that account for thirty-four percent of medication errors, and nine to thirty-seven percent of medication administration has been found to involve at least one error. (Keers, Williams, Cooke, \& Ashcroft, 2013; Mahmood, Chaudhury, \& Valente, 2011; Muñoz et al., 2010; Raban \& Westbrook, 2014; Westbrook et al., 2017) Meanwhile, the research on MAEs has explored medication procedure errors, such as the misidentification of patients and the wrong route of medication administration. These error rates vary from twenty percent to eighty three percent. (Gorini \& Pravettoni, 2013; Mahmood et al., 2011; Raban \& Westbrook, 2014; Westbrook et al., 2017)

To reduce the medication-related errors in healthcare, the World Health Organization (WHO) recommends the six right methods for medication administration, which stands for right medication, right patients, right dose, right route, right time and right documentation. (WHO, 2009) This model is 
accepted globally and it is regarded as a gold standard for medication administration in hospital settings to reduce the human errors of systemic origin in medication procedures. (Australian Commission on Safety and Quality in Health Care, 2012; Joint Commission on Accreditation of Healthcare Organizations, 2013; KARS, 2017; Stein \& Heiss, 2015; Velo \& Minuz, 2009) Regardless, medication errors, including MAEs, still constitute a major problem worldwide. (WHO, 2018) Accordingly, while the use of the six rights is a standard medication procedure worldwide, it is not a panacea for MAEs. (Roughead, Semple, \& Rosenfeld, 2016; WHO, 2009) Several studies of medication adverse events show that a distraction or interruption constitutes only of one of the contributing factors to medication errors. (Biron, Loiselle, \& Lavoie-Tremblay, 2009; Grundgeiger \& Sanderson, 2009)

Interruption or distraction is defined as any attention-disturbing or diverting event or stimulus that causes disengagement from achieving a goal. (Colligan \& Bass, 2012; Pape, 2013) The strong association between interruptions and MAEs is confirmed across a range of studies, and it is now well established that interruptions or distractions during medication administration constitute a leading aetiology of medication errors. (Grundgeiger \& Sanderson, 2009; Reid-Searl \& Happell, 2012) Thus, interventions to reduce interruption or distraction to nurses during medication administration must be developed to circumvent the negative effects of interruptions to the patients, their families, nurses, and to the healthcare system at large. (Roughead \& Semple, 2009) Interruptions to the medication administration (MA) process have been identified as one of the leading causes of medication errors. (ReidSearl \& Happell, 2012) These errors have the potential to have long-term negative effects on the life of the patient, their relatives and the administering nurse, and it could result in financial burdens on the health care system. (Roughead \& Semple, 2009)

Several reviews have examined the effectiveness of interventions to reduce interruptions during medication administration across numerous healthcare settings, including hospitals and aged care. (Hopkinson \& Jennings, 2013; Raban \& Westbrook, 2014; "Strategies to reduce medication errors with reference to older adults.," 2006) These studies identified that a range of interventions could be deployed to reduce nursing distractions during medication administration within the clinical setting. (Hopkinson \& Jennings, 2013; Raban \& Westbrook, 2014; "Strategies to reduce medication errors with reference to older adults.," 2006) Nevertheless, none of the reviews reported that interventions to reduce the distractions during medication administration were effective. In fact, most of the studies demonstrated that the evidence for the effectiveness of interventions to reduce MAEs was weak. (Hopkinson \& Jennings, 2013; Raban \& Westbrook, 2014; "Strategies to reduce medication errors with reference to older adults.," 2006) Accordingly, the suggestion was made to conduct a range of further research to evaluate the effectiveness of MAE interventions, including randomized controlled trials and systematic reviews. (Hopkinson \& Jennings, 2013; Raban \& Westbrook, 2014; "Strategies to reduce medication errors with reference to older adults.," 2006)

Nurses constitute the largest group of health professionals who work in hospital settings and nurses deliver a large proportion of the care provided to patients and their families in the hospital context. Consequently, nursing staff play a pivotal role in ensuring the safety of the patients, which includes medication safety. (Olsen, Goolsby, \& McGinnis, 2009) Therefore, the aim of this review was to examine evidence of the effectiveness of the interventions to reduce the nurses' distractions or interruptions during medication administration and to reduce the error rates related to medication administration and to update the review conducted by Raban and Westbrook in 2012. (Raban \& Westbrook, 2014).

\section{MATERIALS AND METHODS}

This review included studies that were written in English, and the objective of each selected study must address the distractions or interruptions to nurses during medication within the hospital setting. Each of these articles needed to contain the words 'nurse' 'distraction' or 'interruption', 'hospital', 'medication' and 'error'. The exclusion criteria for this review included studies of distractions or interruptions focused on clinicians other than nurses, studies conducted in non-hospital settings, such as aged care, and it also excluded book chapters, editorial content and grey literature.

This review included all studies that reported on the outcomes of an intervention or on a range of interventions that aimed to reduce the nurses' distraction or interruptions during medication administration in a hospital setting. This review considered the studies that reported quantitative data only, including randomized controlled trials, pre- and post-intervention studies that observed and evaluated the distractions to nurses during medication administration or medication administration adverse events. Conference proceedings were excluded from this analysis. This study considered studies that included outcome measures for reducing the nurses' distractions during medication administration and outcome measures for the reduction in medication administration error rates. However, studies that used non-direct observation, such as a self-reported study or other qualitative assessment measures, were excluded.

The purpose of the search strategy was to discover published studies only. This review derived keywords from the research question and used Joanna Briggs' Institute search framework to discover secondary data. The search was conducted in Cinahl, Medline and Embase because the databases provided the required quality of health bibliographies. 
Table 1. Quality appraisal of the included studies

\begin{tabular}{lccccccccccccc}
\hline First Author, year & Q1 & Q2 & Q3 & Q4 & Q5 & Q6 & Q7 & Q8 & Q9 & Q10 & Q11 & Q12 & Q13 \\
\hline Before and after studies & & & & & & & & & & & & & \\
Ching, 2013 & Y & Y & Y & N & Y & Y & Y & Y & Y & - & - & - & - \\
Dall'Oglio, 2017 & Y & Y & Y & N & Y & Y & Y & Y & U & - & - & - & - \\
Flynn, 2016 & Y & Y & Y & Y & Y & Y & Y & Y & U & - & - & - & - \\
Pape, T.M. 2013 & Y & Y & Y & N & Y & Y & Y & Y & Y & - & - & - & - \\
Tomietto, 2012 & Y & Y & Y & N & Y & Y & Y & Y & U & - & - & - & - \\
William, 2014 & Y & Y & Y & N & Y & Y & Y & Y & U & - & - & - & - \\
Cluster Randomized Controlled Study & & & & & & & & & & & & \\
Westbrook, 2017 & Y & Y & Y & Y & Y & U & Y & Y & Y & Y & Y & Y & U \\
\hline
\end{tabular}

Furthermore, the search strategy was limited to literature published between October 2012 and August 2018. The searching time terminated in September 2018 and there was no alert setting in the searched databases in order to engage with the newly published studies. The initial keywords that were used for the search were 'nurses' interruptions', 'nurses' distractions', 'nurses' interference', 'nurses' stopping', 'nurses' 'disturbance', and 'medication administration'. Keywords combined Boolean operators, AND or OR, in the search strings (an example of the final keyword search in Medline can be seen in Appendix 1 on supplementary file).

In this review, the author independently selected both the retrieved titles and abstracts prior to including a full-text appraisal. The retrieval manuscripts were assessed for eligibility in terms of the types of participants, the design of the studies and the outcomes. Furthermore, the Joanna Briggs Institute Meta-Analysis of Statistics Assessment and Review Instrument (JBI-MAStARI) was employed as a critical appraisal instrument to assess the methodological validity of each paper prior to inclusion in this review. During the methodological assessments, several doubts were raised but these doubts were resolved through discussion.

In this review, a standardized data extraction tool from JBI-MAStARI was used to extract the data from the included studies. The data extracted included the study setting, study design, the definition of interruption, the interventions examined, the measured outcomes, the effects of the interventions against the research questions and specific objectives. Data regarding the study settings included the country, the hospitals, the chosen wards or units and the category of the included wards where the research was conducted. The data on the operational definitions of 'interruptions' and 'distractions' was extracted, as was the data regarding the sample size and inter-rater reliability assessment between the observers. The use of the statistical testing was used to measure the outcomes, to compare the participant groups and to evaluate the independence of the data observers or data collectors (Appendix 2). Furthermore, the data that was extracted from the studies included the rates of interruption, the sources of interruption, the completion time of the medication administration, and the rates of medication administration errors. Details of each study selected were also collected, which included the number of medication administration errors that were observed, the number of patients who were administered medication, the number of medication events, the number of observation hours, and the number of observed nurses (Appendix 2). During the data extraction, there were several doubts raised about the extraction methods. However, these issues were resolved through discussions.

The methodological quality of the included study was assessed using JBI MAStARI appraisal instruments. Most of the studies had appropriate sampling with a clear inclusion criterion, a suitable length of follow-up duration, reliable measuring outcomes and appropriate analyses. All of the included studies attained the "Yes" criteria to a minimum of $50 \%$ of the appraisal questions. The assessed studies were considered to have sufficient methodological quality before being included in the review. The results of the quality appraisal of the included studies have been presented in Table 1.

A meta-analysis for this review could not be conducted because of the heterogeneity of the interventions, the interventions' differing durations and the outcome measurements used in the included studies. Therefore, the results of the measured outcomes have been provided via narrative reporting.

\section{RESULTS}

The description of included studies. In this review, three databases were selected and the database search generated 48 articles. From the obtained articles, 14 duplicates were removed and 34 abstracts were screened against the eligibility criteria. From the abstract screening, 15 abstracts were excluded and 19 articles retrieved had a full text. Among the articles, 11 articles were removed either because the interventions were irrelevant to reducing MAEs or because the measurement outcomes were not clearly operationalized. One article was removed because the article did not provide information about the number of units analyzed and the results of the study were presented in percentages, rendering it impossible to compare the study's results with those of other studies. (Aguirre, Wilhelm, Backer, Schoeneman, \& Koehler, 2015) At the end of the selection process, seven studies met the inclusion criteria. The flowchart of the process of inclusion has been depicted in Figure 1. 


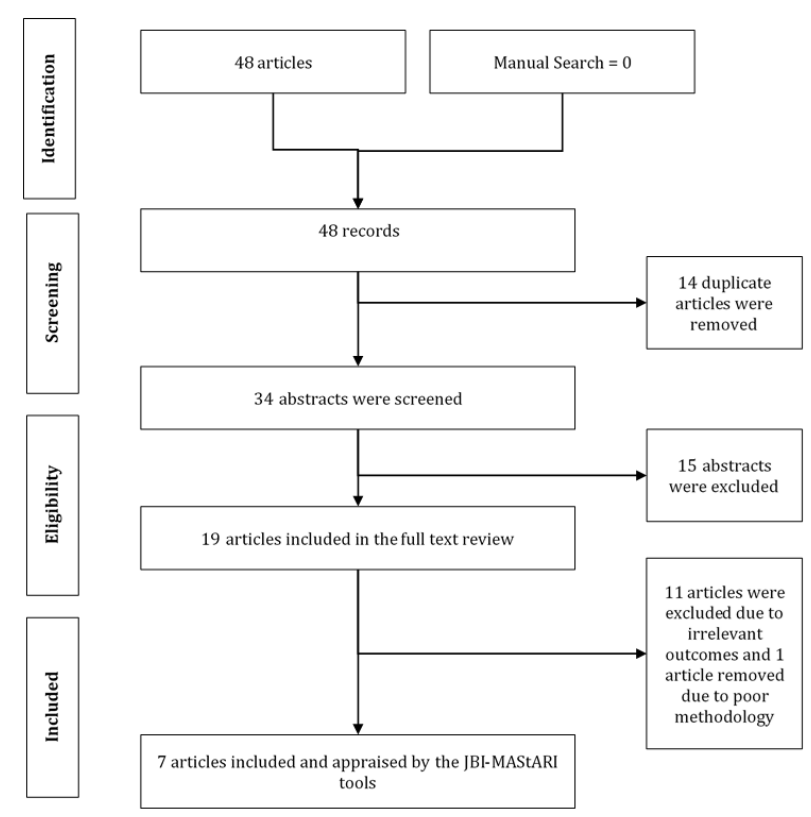

Figure 1. Quality appraisal of the included studies

The characteristics of involved studies. Most of the included studies $(n=4)$ were undertaken in the United States of America (USA), although two were done in Italy and one was conducted in Australia. The majority of the included studies were conducted in hospital wards $(\mathrm{n}=6)$, while one study was completed in a medical centre which was equal to a regional hospital. (Ching, Long, Williams, \& Blackmore, 2013; Dall'Oglio et al., 2017; Flynn, Evanish, Fernald, Hutchinson, \& Lefaiver, 2016; Pape, 2013; Tomietto, Sartor, Mazzocoli, \& Palese, 2012; Westbrook et al., 2017; Williams, King, Thompson, \& Champagne, 2014) The total amount of wards studied was 41 , and the type of ward varied from acute care wards and medical surgical wards through to intensive care wards, medical wards and an emergency unit. (Ching et al., 2013; Dall'Oglio et al., 2017; Flynn et al., 2016; Pape, 2013; Tomietto et al., 2012; Westbrook et al., 2017; Williams et al., 2014).

The majority of the included studies $(n=6)$ assessed the outcomes of the interventions in terms of preventing interruptions to the nurses during medication administration. (Dall'Oglio et al., 2017; Flynn et al., 2016; Pape, 2013; Tomietto et al., 2012; Westbrook et al., 2017; Williams et al., 2014) Furthermore, four studies evaluated the outcome of interventions in terms of eliminating medication errors. (Ching et al., 2013; Flynn et al., 2016; Pape, 2013; Williams et al., 2014) Two studies specified that the aim of their research was to examine the outcome of their interventions on the prevalence of interruption experienced by the nurses and to further scrutinize the multi-tasking rates among nurses during medication administration. (Aguirre et al., 2015; Westbrook et al., 2017) Moreover, the studies were interested in assessing the impact of their selected interventions on the time of medication administration and on the nurses' perceptions of the severity of interruptions and distractions, (Pape, 2013) the strategies used by the nurses to manage any experienced interruptions, (Tomietto et al., 2012) the nurses' perception of the sources of interruption and distraction, the nurses' perceptions of the helpfulness of the interventions and the nurses' compliance with the interventions after they were implemented. (Williams et al., 2014)

The outcome of interest was assessed by the Collaborative Alliance for Nursing Outcomes (CALNOC) Medication Administration Accuracy Assessment in two studies (Ching et al., 2013; Flynn et al., 2016) and three studies utilized the Medication Administration Distraction Observation Sheet (MADOS) to observe the interruption rates. (Dall'Oglio et al., 2017; Pape, 2013; Williams et al., 2014) Westbrook, et al (Westbrook et al., 2017) applied the Work Observational Method by Activity Timing (WOMBAT) Software to collect the outcome of interest in real time and the remaining studies used direct observations for the interruptions. (Tomietto et al., 2012).

Five studies used quasi-experiments with preintervention and post intervention designs with no control groups. The remaining studies employed a quasi-experiment pre-intervention and post intervention within the control group, and there was also a cluster randomized controlled study. Six studies were related to quality improvement projects in the hospital. (Aguirre et al., 2015; Ching et al., 2013; Dall'Oglio et al., 2017; Flynn et al., 2016; Tomietto et al., 2012; Williams et al., 2014) The studies' duration varied from one month to 48 months, which included over 821 nurses who were sampled using a convenience sampling method. (Dall'Oglio et al., 2017; Pape, 2013; Westbrook et al., 2017; Williams et al., 2014) However, three studies did not provide information about the nurses who were included in the studies. (Ching et al., 2013; Flynn et al., 2016; Tomietto et al., 2012).

Five studies specified a definition of interruption. (Dall'Oglio et al., 2017; Flynn et al., 2016; Pape, 2013; Tomietto et al., 2012; Westbrook et al., 2017) One of the studies differentiated between avoidable and unavoidable interruptions, (Flynn et al., 2016) one study defined interruption as being distinct from distraction, (Pape, 2013) while another study made a distinction between medication interruptions and non-medication related interruptions. (Westbrook et al., 2017) Two studies conducted their research without operationally defining the meaning of interruptions in their research. (Ching et al., 2013; Williams et al., 2014) Furthermore, two out of four studies that examined the effectiveness of interventions to limit interruption during medication administration for the purpose of reducing medication errors did not provide a definition of medication errors. (Ching et al., 2013; Pape, 2013).

All of the studies reported that they assessed the occurrence of interruption before the implementation of the interventions and that they compared this baseline measure with the rate of 
interruption after the interventions were implemented. The observations in the studies were of the nurses who worked in the study hospitals. (Ching et al., 2013; Dall'Oglio et al., 2017; Flynn et al., 2016; Westbrook et al., 2017) Two studies did not specify the occupation of the health providers who dispensed the medication, (Pape, 2013; Williams et al., 2014) while one study stated that observations in the study were conducted by the researchers. (Tomietto et al., 2012) Moreover, of the four studies that evaluated the effectiveness of the intervention to limit the interruption during medication administration for the purpose of eliminating medication errors, only two studies provided definitions for the medication errors (Flynn et al., 2016; Westbrook et al., 2017). The remaining studies did not have any definition of the medication errors provided. (Ching et al., 2013; Pape, 2013)

All of the included studies involved direct observation to compare the medication error measurements in the baseline with the medication error measurements in the intervention group, and all observations were conducted by the nurses. Five studies used more than four nurses to gather the data. (Ching et al., 2013; Dall'Oglio et al., 2017; Flynn et al., 2016; Tomietto et al., 2012; Westbrook et al., 2017) The remaining study stated that the research used observers to collect the data, but there was no information provided about the number of observers included. (Pape, 2013; Williams et al., 2014) To evaluate the consistency in judgment during the data collection process, three studies reported that the inter-rater reliability was either $96 \%$ or higher. (Dall'Oglio et al., 2017; Flynn et al., 2016) One study reported a Cohen's Kappa of $>0.90$, (Tomietto et al., 2012) while another study reported that the observers were trained to audit the interventions, although the inter-rater reliability test results were not reported. (Ching et al., 2013) One study reported that the reliability test was not undertaken due to its well established use in a previous study (Pape, 2013), and two remaining studies provided no information about the inter-rater reliability. (Westbrook et al., 2017; Williams et al., 2014).

All of the studies evaluated the implementation of a set of interventions. Four studies evaluated five interventions, (Dall'Oglio et al., 2017; Pape, 2013; Westbrook et al., 2017; Williams et al., 2014) three studies evaluated three interventions (Aguirre et al., 2015; Ching et al., 2013; Tomietto et al., 2012) and one study examined ten interventions. (Flynn et al., 2016) However, most of the studies examined similar interventions (Appendix 5). The interventions included education and workshop sessions for the nurses, education for all health professionals working in the wards, education for the patients, their families and visitors, delineating certain areas as 'quiet zones' 'no interruption zones' or 'medication areas', using conspicuous signs, such as a yellow sash, light sash or a round tabard containing alert text, such as 'do not interrupt', which can be worn by nurses, using indicator lights around medication areas during medication administration, and using check lists of the medication procedures to be carried at all times by nurses when administering medication. Furthermore, diversion strategies during the medication administration included using other members of staff to take emergency calls so then the nurses who were administering medications would not be interrupted. (Flynn et al., 2016; Pape, 2013).

The table in Appendix 7 provides the results of the studies regarding the overall changes in interruption rates after the interventions were implemented. Five studies showed evidence of a reduction in the interruption rates in the post-intervention measurements. (Dall'Oglio et al., 2017; Flynn et al., 2016; Pape, 2013; Westbrook et al., 2017; Williams et al., 2014) Four of these studies demonstrated a statistically significant reduction in the interruption rates, with $\mathrm{p}$ values between 0.0005 and 0.002 , (Dall'Oglio et al., 2017; Flynn et al., 2016; Westbrook et al., 2017; Williams et al., 2014) One study reported an $84 \%$ decrease in interruption rates postintervention. (Pape, 2013) Another study showed that there was a significant increase in interruption rates after the interventions were implemented. (Tomietto et al., 2012) Furthermore, one study examined the effect of the interventions on the time of medication administration and found that the time taken to administer the medication reduced significantly by 1.26 minutes per medication administration. (Pape, 2013) Nevertheless, the study did not provide details about the statistical significance of the change. (Pape, 2013).

Five studies evaluated the change in interruption rates across the different sources (Dall'Oglio et al., 2017; Flynn et al., 2016; Pape, 2013; Tomietto et al., 2012; Westbrook et al., 2017) but only two out of four studies reported that the change was statistically significant. (Dall'Oglio et al., 2017; Tomietto et al., 2012) Dall'Oglio et.al. reported statistically significant reductions in the average interruption occurrences per medication administration event from patients, nursing staff, physicians, phone calls, other personnel, and visitors, but statistically significant reductions in interruption were not reported for interruptions for prescriptions, missing medication, or emergency calls. (Dall'Oglio et al., 2017) Flynn et al's study divided the source of interruption into four different categories, including patient-related interruptions, telephones calls, verbal interactions, and unavailability of resources. The implemented interventions reduced the interruptions caused by telephone calls and verbal or face-to-face interactions, but there were no changes in interruptions due to patient-related issues and resource-related interruptions. (Flynn et al., 2016) This study also reported that the rates of unavoidable and avoidable interruption during medication administration decreased significantly. The study did not provide a description of the sources of the interruptions. (Flynn et al., 2016) Pape's research showed a decrease in interruption rates in the following categories: conversation, health 
professionals, telephone calls, medication issues, urgent telephone call, pager, patients' calls and other interruptions. (Westbrook et al., 2017) Similarly, Tomietto et al's study showed a statistically remarkable reduction in the interruption rates due to the staff members' requests, inadequate amounts of medication and/or materials for medication administration in the trolley, the patients' direct requests, attending to other activities that are not related to the drug round, and answering phone calls from other units. (Tomietto et al., 2012) However, the interventions did not affect the interruption rates due to searching for medication, answering the patients' bells, managing drug documentation, educating the patients' regarding drug administration, and other interruption sources. (Tomietto et al., 2012)

Moreover, Westbrook et al. conducted interventions that were comprised of five 'bundled' elements which were administered across four wards. (Westbrook et al., 2017) These interventions were: (1) the wearing of a 'do not interrupt' medication vest by the nurses during the preparation and administration of medications, (2) interactive workshops with the nurses discussing the purpose of interventions to reduce non-medication-related interruptions and to identify obstacles or aids in medication interventions (such as where to store vests), (3) brief education sessions with nursing and other allied health staff, (4) patient information, which explained why the nurses were wearing a vest and requesting the patients not to interrupt the nurses during medication administration and (5) the use of reminders, such as posters and stickers, to remind the health professionals, other professionals, and patients, et cetera, not to interrupt the nurses during their medication rounds due to safety reasons. (Westbrook et al., 2017) Westbrook et al. found that the bundled interventions were effective at reducing the interruptions from other nurses, patients, telephone calls, families, doctors, call buttons, alarms, allied health professionals, and others. (Westbrook et al., 2017).

Among the included studies, only one study provided a report about the management of interruptions. (Tomietto et al., 2012) The study reported that the implementation of interventions that aimed to limit the interruptions to nurses during medication administration contributed to substantial changes in the attitudes of the nurses regarding interruption management, such that the nurses tended to perceive the changes favorably. (Tomietto et al., 2012) After the interventions, the nurses tended to postpone managing interruptions until near to the end of the medication administration round or they used diversion strategies such as delegating another nurse to manage the interruption. (Tomietto et al., 2012).

Two studies reported direct observation research designs being used to measure medication administration errors, while the remaining studies examined the long-term impact of interventions on medication errors. (Ching et al., 2013; Flynn et al.,
2016) All of the study results demonstrated a decrease in medication administration error rates. Ching et al. reported that improving the layout and design of the medication room, deploying visual signs, and compartmentalization of the nurses' work duties not only decreased the medication error rates but it also reduced safe-practice violations and improved the rate of the correct administration of medication to the patients. (Ching et al., 2013) The medication error types that decreased in the studies included errors due to unavailable drugs, wrong time, wrong dose, wrong route, wrong form and technique, extra doses, and unauthorized drug administration. (Ching et al., 2013) Similarly, Flynn et al. reported that the MAE rates decreased in their study, but the strategies used to reduce the MAEs were conducted together with the interventions to target interruption reduction during medication administration. (Flynn et al., 2016) Both of the studies reported that the reduction in MAE rates were statistically significant across the studies, with p values $<0.001$. (Ching et al., 2013; Flynn et al., 2016).

\section{DISCUSSION}

This systematic review was undertaken to scrutinize the effect of interventions aimed at reducing the interruption to the nurses during medication administration, although the studies involved in this review differed in study design and in the interventions used. There were also discrepancies in the outcomes which led to the necessity of using a narrative analysis. This review included seven studies and compared these studies in terms of study design, outcomes, observers, interventions and outcome measurements. The interventions implemented in the studies were selected to reduce errors during the medication administration, and none of the included studies examined only one intervention. Instead, a package of intervention was used to limit the interruptions to the nurses during the medication administration. (Pape, 2013; Relihan, O’Brien, O’Hara, \& Silke, 2010) A range of previous studies have highlighted that a range of interventions is the most effective at reducing the nursing distractions during medication administration and that these 'bundled' interventions have been reported as effective at reducing medication administration errors. (Pape, 2013; Raban \& Westbrook, 2014; Relihan et al., 2010) However, conclusive evidence from the included studies regarding interruption rates or medication administration error reductions were difficult to establish. This is because of the lack of vigorous study designs in some of the research studies, small sample sizes, and a lack of rigorous statistical analyses. Therefore, the adoption of these interventions into the nursing services in hospital settings should be done with caution, and further studies that use randomized controlled research designs and systematic reviews are required to establish their value before widespread adoption. (Ingham- 
Broomfield, 2016; Murad, Asi, Alsawas, \& Alahdab, 2016)

Generalization across the studies is difficult because the majority of the included studies were conducted in the US and all of the studies were conducted in single hospitals with various wards. Furthermore, inconsistencies in methodology across the studies limit interruptions that can be drawn from their results. (Raban \& Westbrook, 2014) Additionally, five studies showed evidence of reduction in interruption rates both before and after interventions. Four of these studies reported a statistically significant reduction in the MAEs; one study reported a decrease in the interruption rates post-intervention that did not reach statistical significance, while another study demonstrated a significant increase in interruption rates after the interventions were implemented. Only two studies examined the MAE rates and reported statistically significant reductions. Only one study used a controlled research design, which undermined the quality of the results reported. All of the included studies used direct observation outcome measurements. Therefore, the Hawthorne effect must be considered when interpreting the results. (Goodwin et al., 2017; Polit D F and Beck C T, 2018) Most of the data was collected during the week days at the busiest times. This is an important potential confounding factor regarding medication administration (i.e. the data might vary depending on the day of the week and time of day). (Dall'Oglio et al., 2017; Katz, 2010)

The outcome definitions were not clearly defined in several of the studies. The interruption was the main focus but it was not defined in two studies. In the remaining studies, it was divided into several types, including avoidable and unavoidable interruptions and medication and non-medicationrelated interruptions. Similarly, no medication administration errors were defined in the studies that examined the medication administration errors. Therefore, not having an operationalized definition of the outcomes is likely to have affected the empirical measurement of the interruptions and administration errors. (Slife, Wright, \& Yanchar, 2016)

To obtain a comprehensive understanding of the impact of interventions on interruptions during medication administration, it is necessary to know both the sources of interruption and at which stages of medication administration the interruptions occurred. However, the studies that categorized the interruption sources used vague categories of interruption that could obscure the source of the interruption. Only one study examined the occurrences of interruption during the medication administration stages. A lack of information about the interruption rates in each medication stage likely impacted the strength of interventions when it came to reducing the interruptions during medication administration.

The complexities of nursing practices require there to be a continuous availability of nurses to their patients, although the complexity of some tasks means that sometimes it is necessary for the nurses to be undisturbed and isolated from the rest of the ward. This especially applies to medication administration. Therefore, strategies that eliminate interruptions, comparable to the use of a sterile cockpit for airline pilots, need to be adapted into the nursing practice environment. (Hayes, Jackson, Davidson, \& Power, 2015) Nevertheless, Hall et al. argued that it is not possible to entirely eradicate interruptions and distractions from the medication administration process. Therefore, developing a highquality program to safely manage interruptions in the nursing environment is required. (Hayes et al., 2015) Among the included studies, only one study examined the strategies developed by nurses to manage interruptions during medication administration. (Tomietto et al., 2012) However, the studies included in this review failed to look at priority tasks and multitasking related to medication administration. There was also a limitation regarding how the management of interruptions affected emergencies and other necessary interruptions. (Hayes et al., 2015) Therefore, future studies should be conducted to investigate this topic.

It is necessary to note that not all interruptions during the medication administration are negative. Some interruptions are necessary due to medication safety reasons. This topic has been highlighted in previous studies. (Conrad, Fields, McNamara, Cone, \& Atkins, 2010; Raban \& Westbrook, 2014) Conrad et al. argued that defining necessary interruptions is valuable to patient care. Nevertheless, determining 'necessary' interruptions could be challenging due to the potentially subjective interpretation of 'necessary'. (Raban \& Westbrook, 2014) Anthony et al. pointed out that interruptions might be considered important to patient care from one point of view but not from another. (Anthony, Wiencek, Bauer, Daly, \& Anthony, 2010) For example, pager calls might be judged as an unnecessary interruption by the recipient nurse but the call initiator may view the call as a critical and necessary interruption for patient care. In this review, one study reported an interruption as 'necessary' due to medication safety but the study did not provide sufficient data in their report to justify the interruption as 'necessary'. The included studies failed to examine any potential unintended consequences of their interventions. Therefore, this topic should be addressed in future studies to understand any unintended consequences of interruptions during medication administration.

This systematic review had some limitations. The comprehensive search process used in this review used three electronic databases. Therefore some eligible studies might have been missed. Furthermore, this review exclusively included studies that were written in English, therefore this review might have missed highly relevant research that was published in other languages. The studies in this analysis were predominantly conducted in the US, and only one study was conducted in a hospital that 
had several different types of wards. Consequently, the over or under estimation of the interventions aimed to reduce the nursing distractions during the medication administration might have occurred. Additionally, it is important to note that the literature searches, data extraction and data analysis in this review were conducted by the first author and that the second author acted as a reviewer.

\section{CONCLUSION}

This review is an integrative review of the current studies that have used interventions to reduce the interruptions to nurses during medication administration. There was limited evidence of the effectiveness of interventions in terms of either reducing the interruptions to nurses during medication administration or reducing the medication administration error rates. This review summarized the weak evidence of the effectiveness of the interventions aimed at reducing the nurses' interruption during the medication administration and to reduce the medication administration errors. Therefore, the recommendation based on this review is grade B1 (according to JBI Grades of Recommendation), which means that the interventions must be adopted with caution. If the health stakeholders and nurse practitioners adopt these interventions in clinical practice, then their effectiveness needs to be consistently monitored until randomized controlled studies can confirm their clinical value. Future studies with randomized controlled methodologies, adequate sample sizes and a systematic review are all required to examine and explore the effectiveness of the interventions that aim to reduce the interruptions during medication administration to reduce the interruption rates and medication errors overall.

\section{REFERENCES}

Aguirre, T., Wilhelm, S., Backer, S., Schoeneman, S., \& Koehler, A. (2015). Medication Administration Interruptions in a Rural Hospital and Evaluation of a Red Light Intervention. Online Journal of Rural Nursing and Health Care. https://doi.org/10.14574/ojrnhc.v15i2.327

Anthony, K., Wiencek, C., Bauer, C., Daly, B., \& Anthony, M. K. (2010). Safety in intensive care units no interruptions please: Impact of a no interruption zone on medication. Critical Care Nurse. https://doi.org/10.4037/ccn2010473

Australian Commission on Safety and Quality in Health Care. (2012). National Safety and Quality Health Service Standards.

Biron, A. D., Loiselle, C. G., \& Lavoie-Tremblay, M. (2009). Work interruptions and their contribution to medication administration errors: An evidence Review. Worldviews on Evidence-Based Nursing. https://doi.org/10.1111/j.17416787.2009.00151.x

Ching, J. M., Long, C., Williams, B. L., \& Blackmore, C. C. (2013). Using lean to improve medication administration safety: In search of the "perfect dose." Joint Commission Journal on Quality and Patient Safety. https://doi.org/10.1016/s15537250(13)39026-6

Colligan, L., \& Bass, E. J. (2012). Interruption handling strategies during paediatric medication administration. BMJ Quality and Safety. https://doi.org/10.1136/bmjqs-2011-000292

Conrad, C., Fields, W., McNamara, T., Cone, M., \& Atkins, P. (2010). Medication room madness: Calming the chaos. Journal of Nursing Care Quality. https://doi.org/10.1097/NCQ.0b013e3181c3695 d

Dall'Oglio, I., Fiori, M., Di Ciommo, V., Tiozzo, E., Mascolo, R., Bianchi, N., ... Raponi, M. (2017). Effectiveness of an improvement programme to prevent interruptions during medication administration in a paediatric hospital: A preintervention-postintervention study. BMJ Open. https://doi.org/10.1136/bmjopen-2016013285

Flynn, F., Evanish, J. Q., Fernald, J. M., Hutchinson, D. E., \& Lefaiver, C. (2016). Progressive care nurses improving patient safety by limiting interruptions during medication administration. Critical Care Nurse. https://doi.org/10.4037/ccn2016498

Goodwin, M. A., Stange, K. C., Zyzanski, S. J., Crabtree, B. F., Borawski, E. A., \& Flocke, S. A. (2017). The Hawthorne effect in direct observation research with physicians and patients. Journal of Evaluation in Clinical Practice. https://doi.org/10.1111/jep.12781

Gorini, A., \& Pravettoni, G. (2013). Nurses' violations of a medication administration protocol in Italy: an observational study. Clinical Nursing Studies. https://doi.org/10.5430/cns.v1n2p80

Grundgeiger, T., \& Sanderson, P. (2009). Interruptions in healthcare: Theoretical views. International Journal of Medical Informatics. https://doi.org/10.1016/j.ijmedinf.2008.10.001

Hayes, C., Jackson, D., Davidson, P. M., \& Power, T. (2015). Medication errors in hospitals: A literature review of disruptions to nursing practice during medication administration. Journal of Clinical Nursing. https://doi.org/10.1111/jocn.12944

Hopkinson, S. G., \& Jennings, B. M. (2013). Interruptions during nurses' work: A state-of-thescience review. Research in Nursing and Health. https://doi.org/10.1002/nur.21515

Ingham-Broomfield, R. J. (2016). A nurses' guide to the hierarchy of research designs and evidence. Australian Journal of Advanced Nursing.

Joint Commission on Accreditation of Healthcare Organizations. (2013). Joint Commission International accreditation standards for hospitals. Joint Commission Resources.

KARS. (2017). Standar Nasional Akreditasi Rumah Sakit Efektif 1 Januari 2018. Standar Nasional Akreditasi Rumah Sakit.

Katz, M. H. (2010). Evaluating clinical and public health interventions: A practical guide to study 
design and statistics. Evaluating Clinical and Public Health Interventions: A Practical Guide to Study Design and Statistics. https://doi.org/10.1017/CB09780511712074

Keers, R. N., Williams, S. D., Cooke, J., \& Ashcroft, D. M. (2013). Prevalence and nature of medication administration errors in health care settings: A systematic review of direct observational evidence. Annals of Pharmacotherapy. https://doi.org/10.1345/aph.1R147

Mahmood, A., Chaudhury, H., \& Valente, M. (2011). Nurses' perceptions of how physical environment affects medication errors in acute care settings. Applied Nursing Research. https://doi.org/10.1016/j.apnr.2009.08.005

Muñoz, A. B. J., Miguez, A. M., Pérez, M. P. R., Escribano, M. D. V., Garcia, M. E. D., \& Saez, M. S. (2010). Medication error prevalence. International Journal of Health Care Quality Assurance. https://doi.org/10.1108/09526861011029389

Murad, M. H., Asi, N., Alsawas, M., \& Alahdab, F. (2016). New evidence pyramid. Evidence-Based Medicine. https://doi.org/10.1136/ebmed-2016110401

Olsen, L. A., Goolsby, W. A., \& McGinnis, J. M. (2009). Leadership commitments to improve value in health care: Finding common ground: Workshop summary. Leadership Commitments to Improve Value in Health Care: Finding Common Ground: Workshop Summary. https://doi.org/10.17226/11982

Pape, T. M. (2013). The effect of a five-part intervention to decrease omitted medications. Nursing https://doi.org/10.1111/nuf.12025

Picone, D. M., Titler, M. G., Dochterman, J., Shever, L., Kim, T., Abramowitz, P., ... Qin, R. (2008). Predictors of medication errors among elderly hospitalized patients. American Journal of Medical Quality. https://doi.org/10.1177/1062860607313143

Polit D F and Beck C T. (2018). Essentials of nursing research: appraising evidence for nursing practice.

Raban, M. Z., \& Westbrook, J. I. (2014). Are interventions to reduce interruptions and errors during medication administration effective?: A systematic review. BMJ Quality and Safety. https://doi.org/10.1136/bmjqs-2013-002118

Reid-Searl, K., \& Happell, B. (2012). Supervising nursing students administering medication: A perspective from registered nurses. Journal of Clinical Nursing. https://doi.org/10.1111/j.13652702.2011.03976.x

Relihan, E., O’Brien, V., O’Hara, S., \& Silke, B. (2010). The impact of a set of interventions to reduce interruptions and distractions to nurses during medication administration. Quality and Safety in Health Care. https://doi.org/10.1136/qshc.2009.036871

Roughead, E. E., \& Semple, S. J. (2009). Medication safety in acute care in Australia: Where are we now? Part 1: A review of the extent and causes of medication problems 2002-2008. Australia and New Zealand Health Policy. https://doi.org/10.1186/1743-8462-6-18

Roughead, E. E., Semple, S. J., \& Rosenfeld, E. (2016). The extent of medication errors and adverse drug reactions throughout the patient journey in acute care in Australia. International Journal of EvidenceBased Healthcare https://doi.org/10.1097/XEB.000000000000007 5

Slife, B. D., Wright, C. D., \& Yanchar, S. C. (2016). Using operational definitions in research: A bestpractices approach. Journal of Mind and Behavior.

Stein, J. E., \& Heiss, K. (2015). The Swiss cheese model of adverse event occurrence-Closing the holes. Seminars in Pediatric Surgery. https://doi.org/10.1053/j.sempedsurg.2015.08.0 03

Strategies to reduce medication errors with reference to older adults. (2006, June). Nursing Standard (Royal College of Nursing (Great Britain) : 1987). https://doi.org/10.7748/ns.20.41.53.s60

Tomietto, M., Sartor, A., Mazzocoli, E., \& Palese, A. (2012). Paradoxical effects of a hospital-based, multi-intervention programme aimed at reducing medication round interruptions. Journal of Nursing Management. https://doi.org/10.1111/j.13652834.2012.01329.x

Velo, G. P., \& Minuz, P. (2009). Medication errors: Prescribing faults and prescription errors. British Journal of Clinical Pharmacology. https://doi.org/10.1111/j.13652125.2009.03425.x

Westbrook, J. I., Li, L., Hooper, T. D., Raban, M. Z., Middleton, S., \& Lehnbom, E. C. (2017). Effectiveness of a Do not interrupt' bundled intervention to reduce interruptions during medication administration: A cluster randomised controlled feasibility study. BMJ Quality and Safety. https://doi.org/10.1136/bmjqs-2016-006123

WHO. (2009). Patient Safety Curricullum Guide for Medical School.

WHO. (2018). Patient Safety: Data and Statistic.

Williams, T., King, M. W., Thompson, J. A., \& Champagne, M. T. (2014). Implementing Evidence-Based Medication Safety Interventions on a Progressive Care Unit. AJN, American Journal of Nursing. https://doi.org/10.1097/01.naj.0000456433.073 43.7f 